Miami Nature Biotechnology Short Reports

TheScientificWorld (2001) 1(S3), 55SR

ISSN 1532-2246; DOI 10.1100/tsw.2001.169

\title{
MICROSCOPIC PHOTOSENSITIZATION WITH CHLOROMETHYL-X-ROSAMINE ON A SUBPOPULATION OF MITOCHONDRIA INDUCES APOPTOSIS IN HUMAN OSTEOSARCOMA CELLS
}

\author{
Maggie Lum*, Tetsuhiro Minamikawa, and Phillip Nagley \\ Department of Biochemistry and Molecular Biology, PO Box 13D, \\ Monash University, Victoria 3800, Australia \\ * Maggie.Lum@med.monash.edu.au
}

INTRODUCTION. Chloromethyl-X-rosamine (CMXRos; MitoTracker Red) is a widely used fluorescent dye in imaging mitochondria. We have found that it possesses a strong photosensitizing action (1) and induces apoptosis. As a mitochondrially targeted photosensitizer, CMXRos may provide versatile new applications in studies of mitochondrial roles in cell death. We describe the use of a cell biological approach via microscopic photosensitization with CMXRos by confocal laser scanning microscopy (CLSM) to induce damage in a subpopulation of mitochondria that leads to cell death. This will form the basis for investigation of intermitochondrial communication in vivo.

METHOD. Human osteosarcoma 143B TK ${ }^{-}$cells were loaded with $200 \mathrm{nM}$ CMXRos. For imaging and photoirradiation, an inverted CLSM (Leica TCS-NT) with a Leica PL APO 63X/NA 1.2 water immersion objective lens and equipped with a temperature-controlled chamber maintained at $37^{\circ} \mathrm{C}$ was used. Photoirradiation was performed under high intensity scanning conditions: xy-scan mode; speed, 1s/frame; zoom, 4; aperture size, 3.65 airy unit; total of 128 scans; laser power around 200 arbitrary unit on the scale of Leica TCS-NT software. For imaging as such, low intensity scanning was carried out to reduce phototoxicity. Properties of cells and mitochondria were monitored at subsequent time intervals after irradiation. The consequences of CMXRos microscopic photosensitization of a subpopulation of mitochondria in individual cells (partial irradiation) were compared to those of cells with their entire mitochondrial population subjected to photoirradiation (global irradiation). Microscopic photosensitization here involved partial irradiation of about half the mitochondria in individual cells.

RESULTS. Phosphatidylserine (PS) exposure (Annexin V binding) occurred in less than one hour in about $80 \%$ of cells partially irradiated, comparable to that of global irradiation. No uptake of propidium iodide was observed up to $2 \mathrm{~h}$ after partial irradiation, indicating that cells were undergoing early apoptosis, but were not necrotic. However, about $20 \%$ of globally irradiated cells showed PI uptake. Cytochrome $c$ (cyt $c$ ) was released in more than $80 \%$ of partially irradiated cells up to $2 \mathrm{~h}$ after irradiation. Global irradiation showed $60 \%$ of cells to release cyt $c$ before $1 \mathrm{~h}$, and this proportion unexpectedly declined (about twofold) $2 \mathrm{~h}$ after irradiation. This anomaly is discussed below. Some of the cells showed apparent non-release of cyt $c$ with weak punctate staining localized to mitochondria. Following partial irradiation of cells pre-loaded with both CMXRos and rhodamine 123 (Rh123: to monitor mitochondrial 
membrane potential, $\Delta \Psi_{\mathrm{m}}$ ), Rh123 fluorescence declined in both irradiated and non-irradiated mitochondria.

DISCUSSION. The PS exposure and cyt $c$ release during the early stages of apoptosis demonstrate that partial microscopic CMXRos photosensitization induces apoptosis. From PI uptake studies, none of the partially irradiated cells were necrotic in contrast to the presence of a minor population of necrotic cells following global irradiation. This suggests that the progress towards cell death in partially irradiated cells is slower than that in globally irradiated cells. The observations of cyt $c$ release following CMXRos global photoirradiation are anomalous. We propose that this may be due to degradation or conformational changes of cyt $c$ that could avoid immunocytochemical detection, subsequent to its initial release brought about by the severe direct mitochondrial damage (1). The loss of $\Delta \Psi_{\mathrm{m}}$ in the non-irradiated mitochondria and cell death observed following partial irradiation could be attributed to possible communication between the irradiated and non-irradiated mitochondria. Signals could be transmitted from the irradiated to the non-irradiated mitochondria directly through the release of soluble factors or indirectly via a downstream caspase-mediated pathway, currently being tested.

\section{REFERENCE.}

1. Minamikawa, T., Sriratana, A., Williams, D.A., Bowser, D.N., Hill, J.S., and Nagley, P. (1999) J. Cell Sci. 112, 2410-2430 

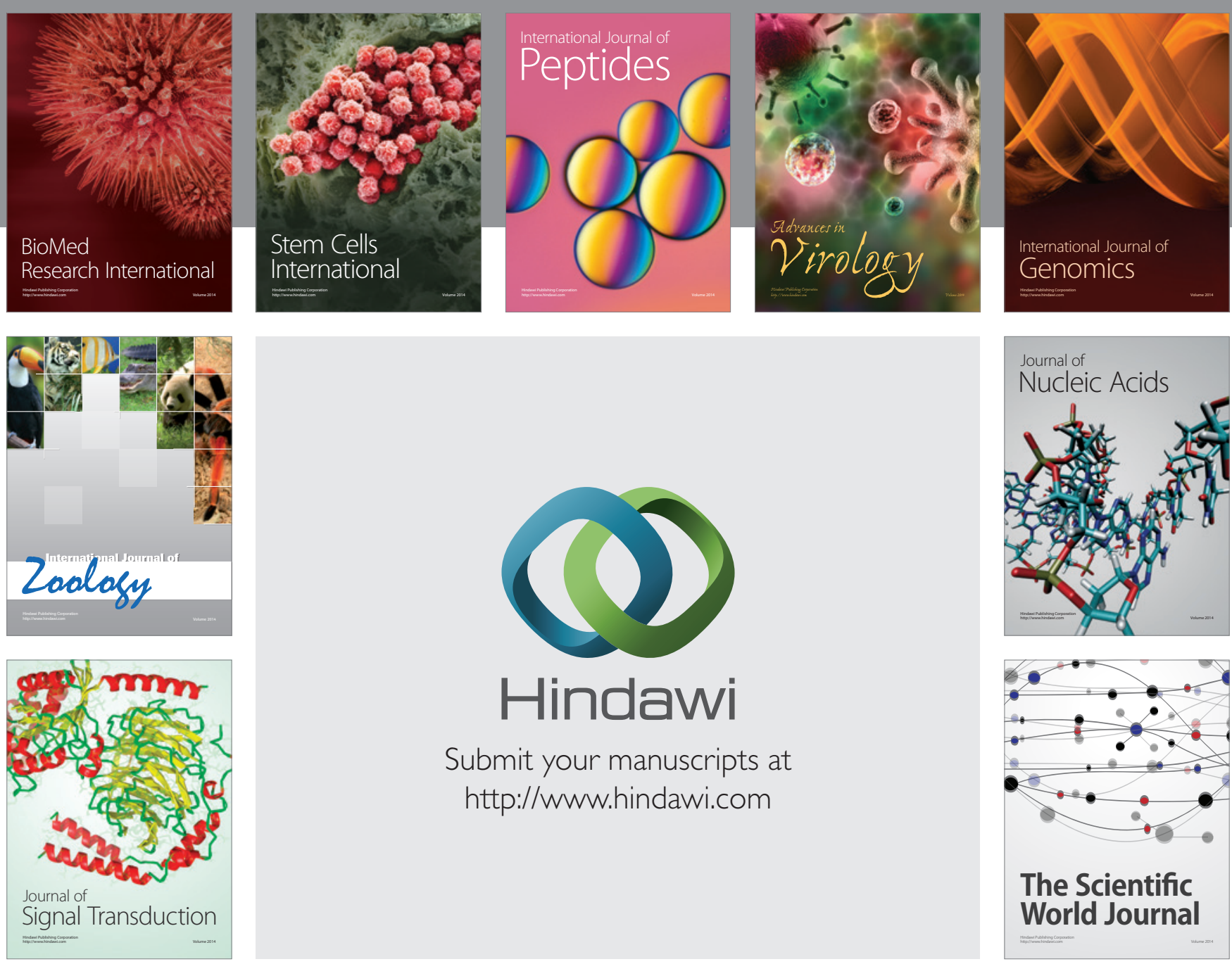

Submit your manuscripts at

http://www.hindawi.com
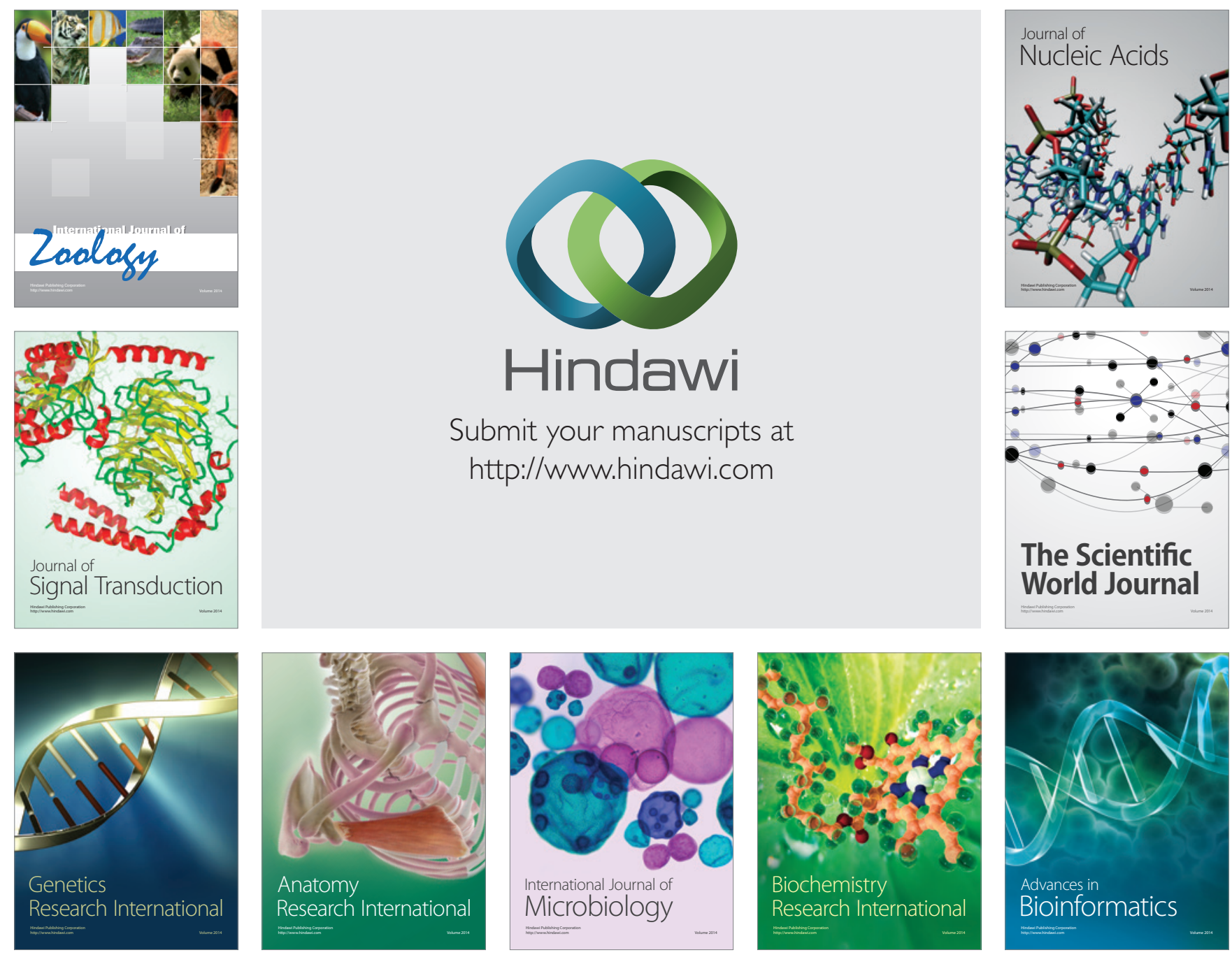

The Scientific World Journal
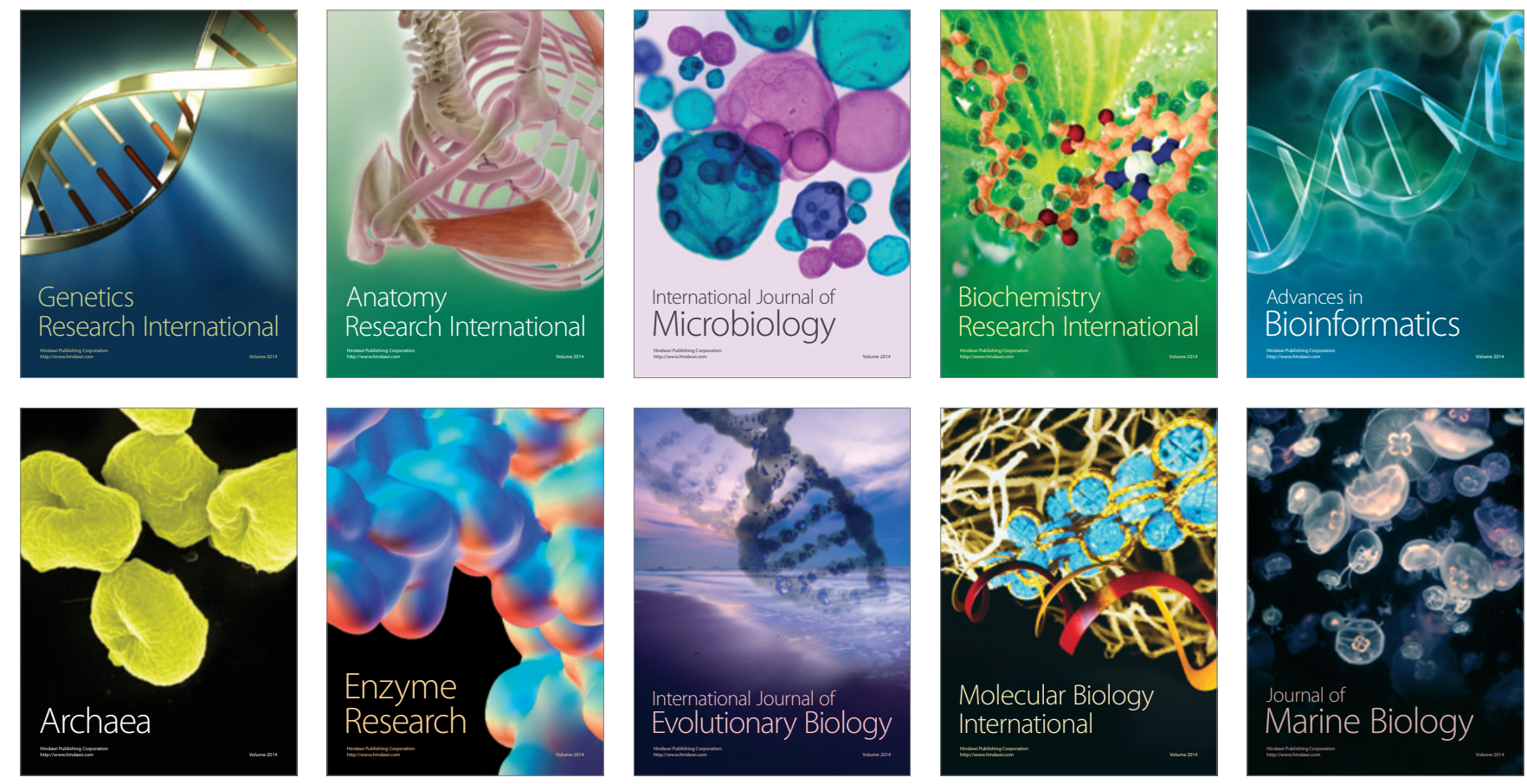